\title{
The Broström-Gould procedure: A case presentation and review of the literature
}

\author{
Michael Finnern*1, John Dupaix ${ }^{2}$, Paul Ryan ${ }^{1}$, Claude Anderson ${ }^{1}$ \\ ${ }^{1}$ Orthopaedic Surgery, Tripler Army Medical Center, Honolulu, United States \\ ${ }^{2}$ Orthopaedic Surgery, The Queen's Medical Center, Honolulu, United States
}

Received: July 11, 2016

DOI: $10.5430 /$ ijdi.v4n1p1
Accepted: August 12, $2016 \quad$ Online Published: August 25, 2016

URL: http://dx.doi.org/10.5430/ijdi.v4n1p1

\begin{abstract}
Lateral ankle sprains are among the most common musculoskeletal injuries in athletes. While most patients recover from lateral ankle sprains, $40 \%$ will have residual symptoms. This case presentation reviews the literature and describes the surgical treatment of a 39-year-old female with a long history of bilateral ankle instability who was treated with the modified Broström-Gould procedure.
\end{abstract}

Key Words: Ankle sprain, Causes, Symptoms, Treatments, Recovery

\section{INTRODUCTION}

Lateral ankle sprains represent one of the most common musculoskeletal injuries. ${ }^{[1,2]}$ Mechanistically, these injuries can arise as a result of forced plantar flexion and inversion causing a progressive loading and failure of the anterior talofibular ligament (ATFL) followed by the calcaneofibular ligament (CFL) and much more rarely the posterior talofibular ligament (PTFL) ${ }^{[3]}$ Ankle instability can also be linked to subtler instability, functional instability, or a combination of all three mechanisms. ${ }^{[4-6]}$ Functional instability arises from deficits in proprioception and muscle function following injury and is typically treated with extensive therapy. ${ }^{[7]}$ Mechanical instability, in turn, arises from laxity of the lateral ankle ligaments secondary to a traumatic injury. Although most of these injuries respond well to conservative treatment with functional rehabilitation, $10 \%-40 \%$ of these individuals will continue to have symptoms despite appropriate conservative care. ${ }^{[8,9]}$ Chronic lateral ankle instability is a diagnosis used to describe a combination of symptoms which can include recurrent sprains, pain with activities, apprehension, and even avoidance of activities. ${ }^{[10]}$ The diagnosis can be made through history and physical examination alone, but imaging modalities are available. The predictive value of stress radiographs has been debated but may provide an additional source of data when the diagnosis is uncertain. ${ }^{[6,11]}$ The American Academy of Orthopaedic Surgeons Orthopaedic Knowledge Online (AAOS OKO) has suggested that a talar tilt of 9 degrees or 3-15 degree difference from the contralateral side may be pathologic. Additionally, an anterior drawer with an absolute value of $10 \mathrm{~mm}$ or $3 \mathrm{~mm}$ difference from the contralateral side may be pathologic. Although non-operative management of chronic ankle instability is an option, certain patients may experience ankle instability and recurrent sprains during their activities of daily living (ADLs) despite appropriate conservative treatment. Surgical reconstruction of the lateral ankle ligaments is indicated in

*Correspondence: Michael Finnern; Email: michael.t.finnern.mil@mail.mil; Address: 4317 Kaikoo Place; Honolulu, HI 96816, United States. 
these patients. ${ }^{[9]}$

\section{Case presentation}

The patient is an otherwise healthy 39 -year-old female with a long history of bilateral ankle instability who was initially injured during a motor vehicle accident wherein a tree branch went through her hind foot. She had multiple prior surgeries for soft tissue coverage and post-operative infections but never had a dedicated surgery performed specifically for ankle laxity. She had failed conservative management to include bracing and therapy. She described instability in both ankles and was unable to run or hike on uneven ground.

On physical examination, she weighed $155 \mathrm{lbs}$, and her height was 5 feet 7 inches. On her right lower extremity, she complained of pain and laxity with talar tilt and anterior drawer stress testing. Slight intrasheath subluxation of peroneals was noted, but she had no pain to palpation over the peroneals. Her sensation was intact to light touch in the saphenous, sural, tibial, superficial and deep peroneal distributions. She had 5/5 motor strength in her extensors, flexors, tibialis anterior, and gastrocnemius-soleus complex. Imaging studies consisted of stress radiographs (see Figure 1). Her talar tilt measured 19 degrees and anterior drawer measured $3.2 \mathrm{~mm}$. An MRI could have been performed to evaluate for other diagnoses but was not needed to evaluate her ligaments as the stress radiographs were consistent with her complaint.

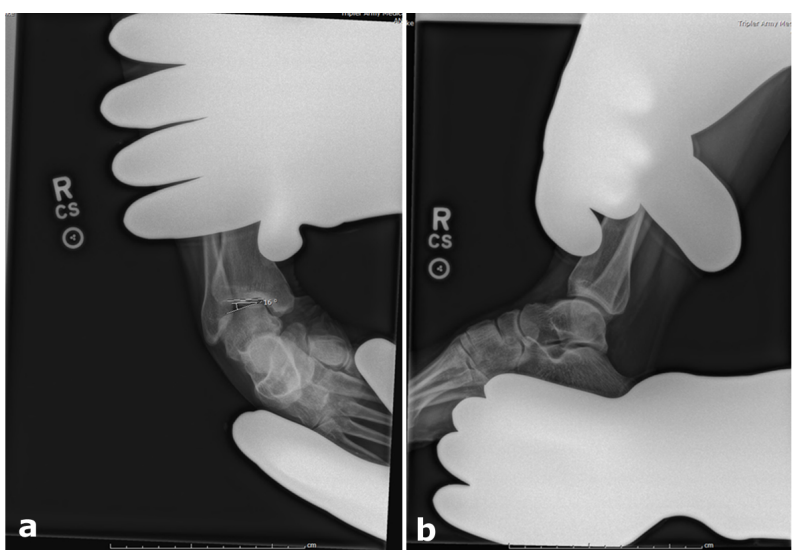

Figure 1. Talar tilt and anterior drawer. Imaging studies consisted of stress radiographs. a. Her talar tilt measured 19 degrees; b. Her anterior drawer was $3.2 \mathrm{~mm}$

This history and physical examination was consistent with chronic symptomatic ankle instability, and a surgical plan was discussed with and agreed upon by the patient.

\section{Operative course}

As an MRI was not performed and chronic ankle instability is associated with a high incidence of intra-articular pathology, a diagnostic ankle arthroscopy was performed prior to the modified Bröstrom-Gould procedure to evaluate the cartilage for impact related injuries. Arthroscopies can be performed at the time of a lateral ligament reconstruction as associated osteochondral defects can be treated during the same anesthetic. ${ }^{[9,18-20]}$

The right ankle was placed in an Arthrex ankle distractor and distracted (see Figure 2) and the surgical anatomy was marked on the skin. The joint was insufflated with $30 \mathrm{ml}$ of sterile saline via the medial portal. This allowed for joint distention which protects the cartilage upon insertion of the trocar. The medial ankle portal was then established with a scalpel for the skin incision. Blunt dissection was then performed to the capsule which was entered with blunt trocar. The lateral portal was then established under direct visualization. Care was taken to incise just the skin and perform blunt dissection down to the capsule in order to protect the superficial peroneal nerve which can cross the surgical field at the lateral portal site. A complete arthroscopic evaluation was performed. There were no areas of chondromalacia on the talar dome or tibial plafond. One of the more important aspects of the evaluation is the dynamic testing of the syndesmosis under arthroscopic visualization as an unstable syndesmosis can mimic a positive anterior drawer in the clinic. In this case, the syndesmosis was stable to external stress examination and anterior translation (see Figure 3a). The ligaments of the syndesmosis, which include the anterior inferior tibiofibular ligament (AITFL), posterior inferior tibiofibular ligament (PITFL) and transverse tibiofibular ligament (TTFL), were readily seen during arthroscopy (see Figure 3a-3c). In this case, the diagnostic arthroscopy was negative for associated injuries.

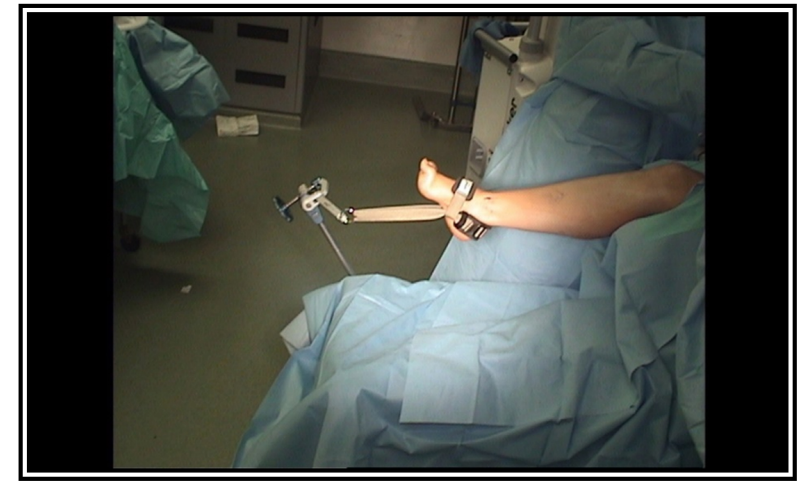

Figure 2. Distraction. Non-invasive ankle distraction 

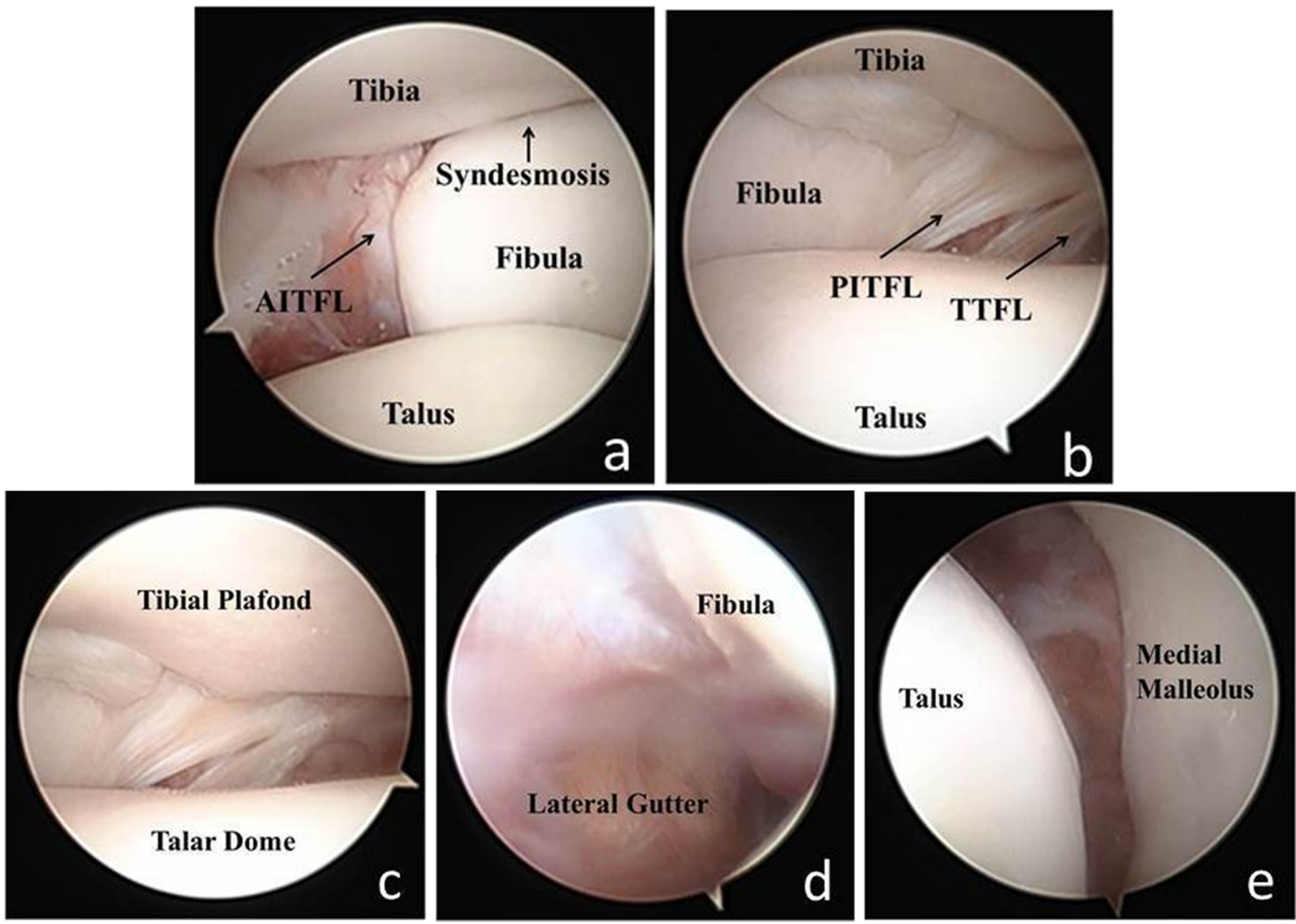

Figure 3. Portal views. a. Stable syndesmosis under external stress evaluation; b. Posterior inferior tibiofibular ligament and transverse tibiofibular ligament; c. Talar dome and tibial plafond; d. Lateral gutter; e. Medial gutter

Attention was then turned to the lateral ankle. The distractor was removed and the leg was left in the leg holder which allowed excellent access to the lateral aspect of the ankle. A curvilinear incision was made which began just posterior to the fibula and extended across the inferior fibula in line with the fourth metatarsal (see Figure 4). Once a full thickness incision was made, the deeper dissection began in the superior aspect of the wound and was carried down to the peroneal sheath. The sheath was not opened at this point. Staying close to the peroneal sheath and fibula periosteum, a superficial flap was dissected from the deep fascia with blunt dissection. This flap should protect the superficial peroneal nerve although the nerve is not visualized within the dissection. The flap was raised to the level of the lateral portal. Once the flap was complete, the peroneal sheath was exposed and opened. Typically, the sheath only needs to be opened for a distance of $1 \mathrm{~cm}$, but in this particular case, the sheath was opened to allow for visualization of the peroneal tendons which were evaluated and found to be intact. The peroneal tendons were then protected with a Davis retractor as a Kelly forceps was placed into the lateral capsular portal and then passed deep to the capsuloligamentous complex which included the ATFL. The tip of the Kelly must be seen exiting the peroneal sheath. With the peroneal tendons protected, the capsuloligamentous complex was then sharply divided over the top of the Kelly. The lateral aspect of the talus should be seen within the wound. The CFL often requires a separate division if included in the repair. Once the deep dissection over the Kelly was complete, it was straightforward to separate the inferior extensor retinaculum from the deeper capsule and ligaments. The superior edge of the capsuloligamentous complex was partially freed from the lateral malleolus with electrocautery in order to ensure that sutures included the full thickness of the flap. Zero Ethibond sutures were then passed into the capsuloligamentous complex in a vest over pants fashion while protecting the peroneal tendons (see Figure 5). The extensor retinacular layer was used to augment the repair with 0 Vicryl and the peroneal sheath closed with the same suture. A post-operative splint was applied with the ankle in neutral dorsiflexion.

She presented for follow-up and suture removal at postoperative day \#14. She remained sensate to light touch in superficial peroneal, deep peroneal, saphenous, sural and tibial nerve distributions. She remained motor intact. At that point, she was placed in a short leg cast walking cast for a period of four weeks. At her routine six week follow-up appointment, she was placed in a controlled ankle walker boot and referred to physical therapy. At one year follow-up, 
she had mild discomfort at the surgical site but reported resolution of her instability and stated she would undergo the same procedure again.

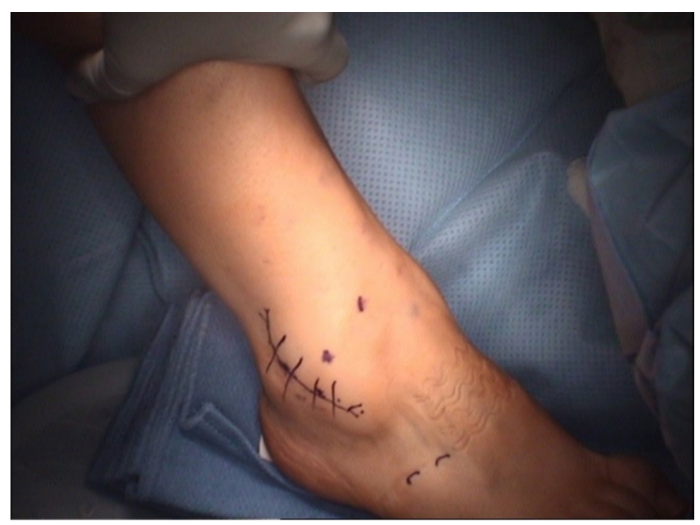

Figure 4. Anatomy marking. Lateral skin incision

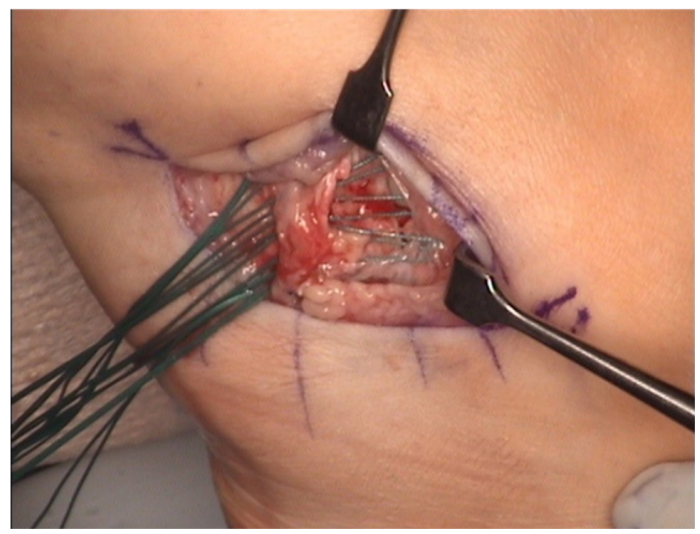

Figure 5. Capsuloligamentous suture placement. Ethibond sutures placed in capsuloligamentous complex in vest over pants configuration

\section{Discussion}

Surgical management of chronic ankle instability has evolved over the last 100 years. In 1932, Nilsonne et al. described the use of the peroneal brevis in order to reconstruct a patient with chronic instability. ${ }^{[21]}$ At about the same time, Elmsie et al. described the use of fascia lata. ${ }^{[22]}$ Tenodesis techniques remained the clinical standard in the literature until the 1960s. ${ }^{[23,24]}$ In 1966, Broström described various anatomic repairs of the native ligaments for both acute and chronic injuries. ${ }^{[3,13]}$ The addition of the inferior retinaculum was described in 1980 by Dr. Nathaniel Gould. ${ }^{[14]}$ The success of the Broström procedure has been well described with the majority of studies reporting $87 \%-100 \%$ success. ${ }^{[9,13-15]}$ In a cadaveric study comparing the modified Broström-Gould procedure to tendon reconstruction procedures, Liu et al. found that the anatomic repair technique resulted in less loss of motion in addition to greater mechanical restraint. ${ }^{[17]}$ In a retrospective review of 54 patients, Krips et al. found that the modified Broström-Gould procedure was superior to tendon reconstructive procedures in terms of final stability as determined through stress radiographs and clinical outcomes. ${ }^{[25]}$ While all surgical treatments should be tailored to the individual patient, the modified Broström-Gould procedure restores the native anatomy, has demonstrated efficacy both in the lab and in the clinic, and should be one of the procedures in the armamentarium of orthopaedic surgeons who treat lateral ankle instability.

\section{CONFLicts OF InTEREST Disclosure}

The authors have declared no conflicts of interest.

\section{REFERENCES}

[1] Bridgman SA, Clement D, Downing A, et al. Population based epidemiology of ankle sprains attending accident and emergency units in the West Midlands of England, and a survey of UK practice for severe ankle sprains. Emerg Med J. 2003; 20: 508e10.

[2] Colville MR. Surgical treatment of the unstable ankle. JAAOS. 1998; 6: $368-377$

[3] Broström L. Sprained ankles: I. Anatomic lesions in recent sprains. Acta Chir Scand. 1964; 128: 483-495. http://dx.doi.org/10. 5435/00124635-199811000-00005

[4] Kannus P, Renstrom P. Current concepts review. Treatment for acute tears of the lateral ligaments of the ankle. Operation cast or early controlled mobilization. J Bone. Joint Surg. 1991; 73A: 305e12.

[5] Bosien WR, Staples OS, Russel SW. Residual disability following acute ankle sprains. J Bone Joint Surg Am. 1955; 37: 1237-43. PMid:13271470
[6] Rubin G, Witten M. The Talar-Tilt Angle and the Fibular Collateral Ligaments: A Method for the Determination of Talar Tilt. JBJS. 1960: 42(A): $311-326$

[7] Wester JU, Jespersen SM, Nielsen KD. Wobble Board Training After Partial Sprains of the Lateral Ligament of the Ankle: A Prospective Randomized Study. J Ortho Sports Phys Ther. 1996; 23: 332-6.

[8] Hiller CE, Kilbreath SL, Refshauge KM. Chronic ankle instability: evolution of the model. J Athl Train. 2011; 46: 133-41. PMid:21391798 http://dx.doi.org/10.4085/1062-6050-46. 2.133

[9] Karlsson J, Lasinger O. Lateral instability of the ankle joint. Clin Orthop. 1992; 276: 253e61.

[10] Guillo S, Bauer T, Lee JW, et al. Consensus in chronic ankle instability: aetiology, assessment, surgical indications and place for arthroscopy. Orthop Traumatol Surg Res. 2013; 99(8 Suppl): S411-9. PMid:24268842 http://dx.doi.org/10.1016/j.otsr. 2013.10.009 
[11] Grace DL. Lateral Ankle Ligament Injuries. Inversion and Anterior Stress Radiography. Clinical Orthopedics and Related Research. 1984; 183: 153-9. PMid:6697582

[12] Kobayashi T, Gamada K. Lateral Ankle Sprain and Chronic Ankle Instability: A Critical Review. Foot Ankle Spec. 2014; 7(4): 298-326.

[13] Broström L. Sprained ankles: VI. Surgical treatment of "chronic" ligament ruptures. Acta Chir Sand. 1966: 132: 551-565. PMid:5339635

[14] Gould N, Seligson, Gassman J. Early and late repair of lateral ligament of the ankle. Foot \& Ankle.1980; 1(2): 84-89. http: //dx.doi.org/10.1177/107110078000100206

[15] Hamilton WG, Thompson FM, Snow SW. Modified Broström procedure of lateral ankle instability. Foot \& Ankle. 1993; 14: 1-7. http://dx.doi.org/10.1177/107110079301400101

[16] Karlsson J, Bergstein T, Lansinger O, et al. Reconstruction of the lateral ligaments of the ankle for chronic lateral instability. JBJS Am. 1988; 70: 581-588. PMid:3356725

[17] Liu S, Baker C. Comparison of Lateral Ankle Ligamentous Reconstruction Procedures. AJSM. 1994; 22(3): 313-17. http://dx.doi .org/10.1177/036354659402200303

[18] Kerr HL, Bayley E, Jackson R, et al. The role of arthroscopy in the treatment of functional instability of the ankle. Foot Ankle Surg. 2013; 19(4): 273-5. PMid:24095237 http://dx.doi.org/10.10 $16 / j$. fas. 2013.06 .008
[19] Takao M, Ochi M, Oae K, et al. Diagnosis of a tear of the tibiofibular syndesmosis. The role of arthroscopy of the ankle. J Bone Joint Surg Br. 2003; 85(3): 324-9. PMid:12729102 http://dx .doi .org/10. 1302/0301-620X.85B3.13174

[20] Choi WJ, Lee JW, Han SH, et al. Chronic lateral ankle instability The effect of intra-articular lesions on clinical outcome. Am J Sports Med. 2008; 36: 2167-72. PMid:18669983 http://dx.doi.org/1 $0.1177 / 0363546508319050$

[21] Nilsonne H. Making a New Ligament in Ankle Sprain. JBJS Am. 1932: 14A. 380-1.

[22] Elmslie RC. Recurrent Subluxation of the Ankle Joint. Annals of Surgery. 1934; 100: 64-7. http://dx.doi.org/10.1097/00000 658-193408000-00012

[23] Watson-Jones R. Fractures and Joint Injuries. 4th Ed. Vol II. Edinburgh: E\&S Livingstone Ltd. 1955: 821-3.

[24] Evans DL. Recurrent Dislocation of the Ankle: A Method of Surgical Treatment. Proc. R. Soc. Med. 1953; 46: 343-348. PMid:13055916

[25] Krips R, VanDijk CN, Halasi T, et al. Long Term Outcomes of Anatomical Reconstruction versus Tenodesis for the Treatment of Chronic Anterolateral Instability of the Ankle Joint: A Multicenter Study. Foot Ankle Int. 2001; 22: 415-421. PMid:11428761 